\title{
Ice cold carbonated water: a therapy for persistent hyperawareness of pharyngeal mucus and throat clearing
}

\author{
A N Acharya, S Mirza, N S Jones
}

\begin{abstract}
Chronic throat clearing or a feeling of 'something' at the back of the oropharynx or nasopharynx is a common cause for referral to otorhinolaryngology services. While treatment of an underlying causative condition might be expected to improve these symptoms, in many cases a clear underlying cause is not found. Currently, there is no recognized treatment which is effective against these troublesome symptoms. This observational study investigated the effectiveness of a regime of sipping ice cold carbonated water to try to break the vicious cycle of throat clearing. Seventy-two patients with these symptoms who had previously been advised to use the regime were contacted with a postal questionnaire. Sixty-three per cent of patients documented an improvement in their symptom severity score. The most severely and most frequently affected patients had the greatest benefit. We conclude that the suggested regime can be effective in breaking the vicious cycle of persistent throat-clearing.
\end{abstract}

Key words: Pharynx; Mucus; Water; Drinking

\section{Introduction}

Chronic throat clearing or a feeling of 'something' at the back of the oropharynx or nasopharynx is a common cause for referral to out-patient otorhinolaryngology services. This symptom has a number of postulated aetiologies. Commonly reported causes include post-nasal drip syndrome, ${ }_{1}^{1}$ gastroesophageal reflux disease, ${ }^{2}$ laryngopharyngeal reflux ${ }^{3}$ and asthma. ${ }^{4}$ Chronic throat clearing is rarely due to disorders such as a vocal tic, presenting in childhood in neuropsychiatric disorders such as Tourette syndrome. ${ }^{5}$ Other individually reported causes include psychogenic throat clearing, ${ }^{6}$ a sensory neuropathy involving the superior or recurrent laryngeal nerves, ${ }^{7}$ and ascariasis. ${ }^{8}$

The differential diagnosis of nasal discharge includes: persistent or intermittent allergic rhinitis; idiopathic rhinitis; post-infectious rhinitis; chronic rhinosinusitis; allergic fungal sinusitis; environmental irritants; and rhinitis associated with pregnancy. ${ }^{9}$ While the term 'drip' in post-nasal drip syndrome implies that mucus can drip from the soft palate or nasopharynx into the larynx, this does not happen because of the rheological properties of mucus, namely, its cohesional forces, spinnability and adhesiveness. ${ }^{10}$ If mucus becomes infected, its viscosity increases and its visco-elastic properties make it even less liable to drip. ${ }^{11}$ Therefore the concept of mucus 'dripping' from the nasopharynx and thereby inducing a persistent requirement for the patient to clear their throat is unlikely. If the cause of chronic throat clearing is truly nasal disease, then a more likely mechanism is global inflammation of 'one airway' ${ }^{12}$ as manifest by the coexistence of rhinitis and asthma in patients. ${ }^{13}$ Only a small proportion of patients with all forms of rhinitis complain of post-nasal mucus or throat clearing. Inflammatory mediators in the lower airways are raised in postnasal drip syndrome, cough variant asthma and gastroesophageal reflux disease, supporting the theory of 'one airway' being involved. ${ }^{12}$ It has been proposed that these mechanisms could explain why asthma ${ }^{4}$ and laryngopharyngeal reflux ${ }^{3}$ might cause some patients to repeatedly clear their throat.

Koufman et $\mathrm{al}^{3}{ }^{3}$ suggested that laryngopharyngeal reflux induces chronic throat clearing due to the development of an inflammatory condition of the pharyngeal mucosa. While a few small studies have supported this hypothesis, Sen et al., ${ }^{14}$ in a systematic review to assess the efficacy of proton pump inhibitors in the treatment of laryngopharyngeal reflux, found that, at that time, there was not enough evidence in the literature to support treatment with proton pump inhibitors.

Pharyngeal mucosal inflammation of any aetiology, local or more distant, may have the potential to cause chronic throat clearing. Throat clearing

From the Department of Otorhinolaryngology - Head and Neck Surgery, Queens Medical Centre University Hospital, Nottingham, UK.

Accepted for publication: 14 July 2006. 
may then contribute to a vicious cycle in which the process of clearing the throat leads to a hypersensitivity of the pharyngeal mucosa, resulting in a hyperawareness of normal pharyngeal mucus or a foreign body sensation. Throat clearing and hawking is utilized as a remedial tactic but this appears to exacerbate the patients' symptoms.

Treatment of a possible underlying causative condition (such as rhinosinusitis, asthma or laryngopharyngeal reflux) might be expected to break the cycle, allowing the tissues an opportunity to settle and the problem of a persistent need to clear one's throat to be resolved. However, in many cases, a clear underlying cause for the symptoms is not found and intervention directed at these is often disappointing. While patients are often discharged from clinic with the reassurance that there is no evidence for pathology, there is currently no recognized treatment which is effective against these troublesome symptoms. Persistent throat clearing is known to significantly reduce patients' health-related quality of life, with a particularly dramatic effect on social function. ${ }^{15}$ The negative psychological impact this has on patients may contribute to the vicious cycle by inappropriately focusing patients' attention on their symptoms such that they attach an excessive significance to them.

This observational study aimed to investigate the effectiveness of an empirical regime that has anecdotally been found to help patients, i.e. sipping ice cold carbonated water, in order to try to break the cycle of throat clearing.

\section{Material and methods}

Data were analysed retrospectively for 72 patients who had presented with chronic throat clearing, either in isolation or associated with other symptoms, between December 2000 and March 2005 (52 months) to a specialist rhinology clinic. Symptoms and signs of an underlying pathology, including rhinosinusitis, asthma, gastroesophageal reflux disease and laryngopharyngeal reflux, had been enquired about and taken into consideration. Patients had been advised that their symptoms may have been exacerbated by a hyperawareness of their pharyngeal mucus. The vicious cycle described earlier had been explained and the importance of breaking this cycle had been emphasized.

Each patient had been instructed to acquire carbonated water, maintaining at least two large bottles at any one time. They were to place the bottles in the freezer to allow ice crystals to form, thereafter transferring them to the refrigerator. The patients were to keep one bottle on their person at all times, drinking some of the ice cold carbonated water on each occasion they felt a need to clear their throat. The postulated mechanism of action was that the ice cold carbonated water would stimulate the oropharynx and hypopharynx and, as far as the patient was concerned, remove any sensation of mucus which might be present. Once the ice crystals in the bottle in use had disappeared, the patient had been instructed to return the bottle to the freezer and to use the second bottle from the refrigerator. Alternating the bottles in this way had maintained the cold temperature of the water being consumed. The patients had been instructed to strictly adhere to this therapy for four continuous days and nights, particularly in the morning if they had previously tried to hawk up their mucus.

The patients identified for the study were contacted by post with a single-sided A4 questionnaire, accompanied by a covering letter describing the purpose of the study and a stamped, addressed envelope with which to return the completed questionnaire. The questionnaire categorized the patient's severity of symptoms (i.e. 'never', 'mild', 'moderate', 'severe' or 'very severe') and the frequency with which they occurred (i.e. 'every few seconds', 'every few minutes', 'every hour', 'every few hours' or 'every few days') before and after the treatment. Patients were asked to quantify the subjectively perceived effectiveness of the therapy (i.e. 'didn't help at all', 'helped a little', 'helped some', 'helped considerably' or 'cured'). Finally, enquiries were made about the presence of specific additional symptoms and which of these, if any, resolved with the treatment.

\section{Results and analysis}

The response was 69 per cent from two mailings. The remaining non-responders were then contacted by telephone. Of the 72 patients identified and contacted, three had moved residence and no forwarding address was available, three were sceptical and hadn't tried the recommended therapy, and one claimed not to have been advised of the therapy at their consultation. One patient had since died of an unrelated pathology. Complete data were therefore available for 64 patients. Of these, 32 were female and 32 were male. Their mean age was 49 years (range 22-83 years) and the mean duration of symptoms at initial consultation had been 52 months (range 12-360 months). The mean follow up was 16 months.

Before therapy, the mean and median severity of symptoms had been 'severe' (range, 'moderate' to 'severe') (Figure 1). The mean frequency of symptoms had been 'every few minutes' to 'every hour'

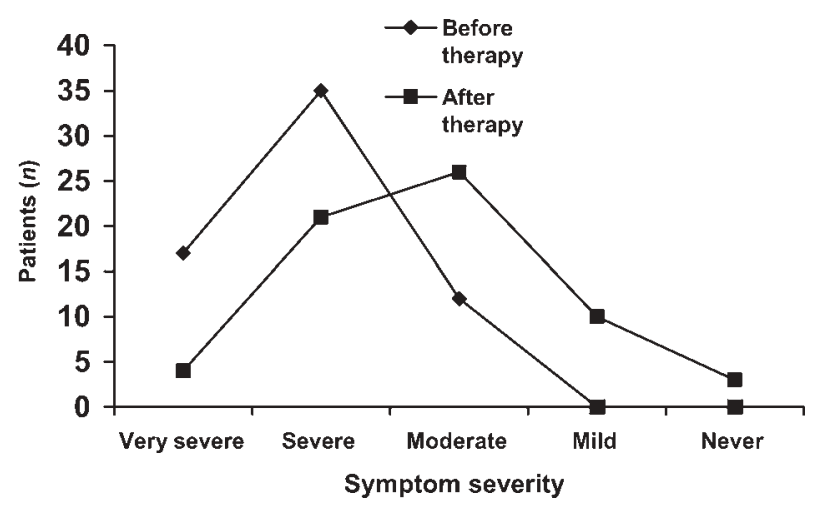

FIG. 1

Severity of symptoms before and after therapy. 
(range, 'every few seconds' to 'every few days') and the median frequency of symptoms had been 'every few minutes' (Figure 2). The severity and frequency assessment tool was then quantified by assigning a value of one to five, in order of increasing severity or frequency, and a symptom severity score was calculated by multiplying these together. The mean symptom severity score before therapy was 12.8 . Additional symptoms experienced had included the feeling of mucus in the throat $(51 / 64,80$ per cent), excessive phlegm or mucus (41/64, 64 per cent), hoarseness $(31 / 64,48$ per cent $)$, heartburn (24/64, 38 per cent), and a cough present for more than one month $(22 / 64,34$ per cent).

Overall, 63 per cent of patients demonstrated an improvement in their symptom score. The average symptom severity score improved from 12.8 to 8.4 , with the most severely and most frequently affected patients having the greatest benefit. Fifty-three per cent of patients felt that the severity of their symptoms had improved with therapy; the median categorization of severity improved from 'severe' to 'moderate' (Figure 1). Forty-eight per cent of patients felt that the frequency of their symptoms had improved with therapy; the median categorization of frequency improved from 'every few minutes' to 'every hour'. Ten patients (16 per cent) experienced symptoms 'every few days' or less and three patients (5 per cent) were cured of their symptoms altogether. The mean and median subjective perception by the patients of the overall effectiveness of the therapy was that it had 'helped'.

With the exception of heartburn, all the additional symptoms patients experienced also improved with the therapy (Figure 3). Twenty-four of the 64 patients (38 per cent) described heartburn as an additional symptom (these patients were termed the gastroesophageal reflux disease group). This group of patients perceived the therapy to be less effective than did those patients who did not experience heartburn as an additional symptom (termed the non-gastroesophageal reflux disease group). The improvements seen in the additional symptoms investigated were more dramatic in the nongastroesophageal reflux disease group than in the

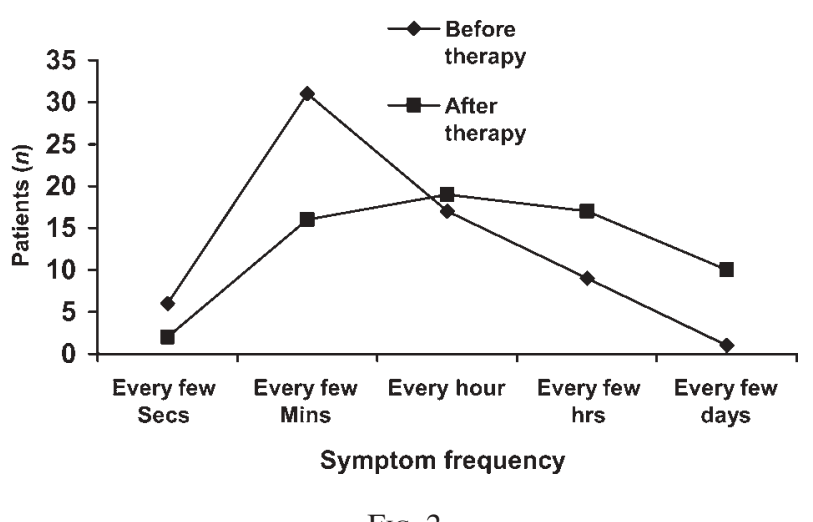

FIG. 2

Frequency of symptoms before and after therapy. Secs $=$ seconds; $\operatorname{mins}=$ minutes; hrs $=$ hours

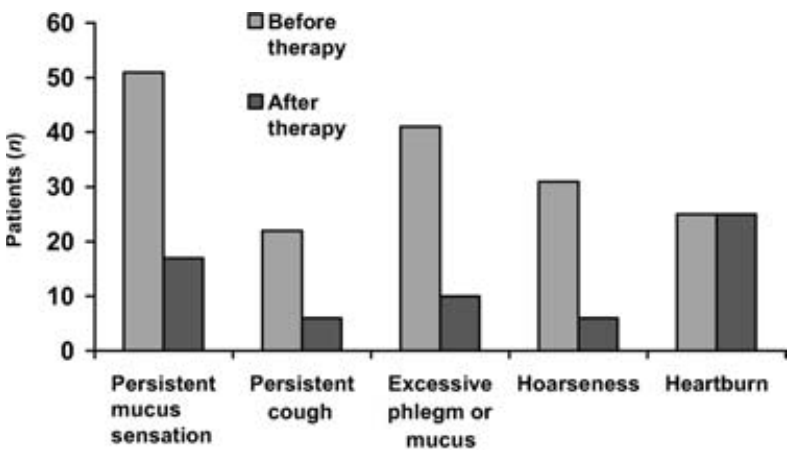

FIG. 3

Additional symptoms before and after therapy.

gastroesophageal reflux disease group: 41 per cent resolution of a persistent sensation of mucus in the throat compared with 18 per cent, 38 per cent resolution of chronic cough compared with 0 per cent, and 27 per cent resolution of hoarseness compared with 13 per cent, respectively. These results suggest that, in patients in whom the reflux of gastric contents into the hypopharynx occured in volumes sufficient to cause symptoms of heartburn (in contrast with laryngopharyngeal reflux, in which the volume is insufficient to cause these symptoms), this persistent source of mucosal insult reduced any beneficial effect the ice cold carbonated water therapy may have conferred.

\section{Discussion}

The findings of this study suggest that sipping ice cold carbonated water may be effective in breaking the vicious cycle of repetitive throat clearing which patients who present with this symptom often complain of. The mechanism of action is open to debate. In some patients, resolution of any hypertonicity of musculature in the hypopharynx and laryngeal inlet may be the principal effect. In other cases in which patients demonstrate somatization, addressing underlying psychogenic components, particularly depression and anxiety, may play a more significant role. In these cases, it is important to minimize the number of investigations, withdraw unnecessary treatment, address any psychological problems and, whilst fully acknowledging the patient's symptoms, to give unambiguous reassurance. ${ }^{16} \mathrm{~A}$ modest improvement in the patient's symptoms achieved by these means might result in a more dramatic improvement in patients' ability to function. Distraction of these patients from their symptoms may contribute to their rehabilitation and encourage recovery from the psychogenic vicious cycle they appear to have developed. A third mechanism of action might be a placebo effect or cognitive dissonance. $^{17}$

We believe that, rather than acting in isolation, the demonstrated positive effects of this therapy reflect a combination of these mechanisms, as the symptoms concerned are usually due to a combination of the proposed pathologies - organic and psychogenic in varying proportions. This is supported by the 
variety of symptoms with which these patients often present.

The advantages of this treatment are that it is cheap, easily initiated and free of side effects. The regime is straightforward to explain and easily understood by patients.

The limitations of this study should be appreciated. Firstly, as an observational study, the findings provide no more than an insight into a potential treatment for a problem which is commonly encountered in otorhinolaryngology. Secondly, patient bias may have influenced the findings; patients who noticed some improvement in their condition might have over-exaggerated the effect of the treatment, while dissatisfied patients might have overlooked small improvements. Therefore a case-control study is required to produce results which can be validated statistically, before one can draw any significant conclusions.

- Chronic throat clearing is a common presenting problem to ENT services and is known to significantly reduce patients' health-related quality of life

- Throat clearing may contribute to a vicious cycle in which the process of clearing the throat leads to a hypersensitivity of the pharyngeal mucosa

- When an underlying causative condition can be identified, treatment aimed at this may alleviate the symptoms

- An underlying cause for the symptoms is often not found, and there is currently no recognized therapy which is effective at reducing the burden of symptoms

- A regime of sipping ice cold carbonated water over four days and four nights whenever patients have a sensation of mucus or want to clear their throat can be effective in breaking the vicious cycle of persistent throat clearing and in improving their symptoms

\section{Conclusion}

The suggested regime, of sipping ice cold carbonated water over four days and four nights whenever patients have a sensation of mucus or want to hawk or clear their throat, can be effective in breaking the vicious cycle of persistent throat clearing. However, before considering this therapy, clinicians must exclude recognized causes of chronic throat clearing. A thorough explanation to the patient of the physiology of mucus production is necessary to promote their understanding of the mechanism of action and to improve their compliance with the treatment.

\section{References}

1 Irwin RS, Pratter MR, Holland PS, Corwin RW, Hughes JP. Postnasal drip causes cough and is associated with reversible upper airway obstruction. Chest 1984;85:346-52

2 Ward PH, Berci G. Observations on the pathogenesis of chronic non-specific pharyngitis and laryngitis. Laryngoscope 1982;92:1377-82

3 Koufman JA. The otolaryngologic manifestations of gastroesophageal reflux disease (GERD): a clinical investigation of 225 patients using ambulatory 24-hour $\mathrm{pH}$ monitoring and an experimental investigation of the role of acid and pepsin in the development of laryngeal injury. Laryngoscope 1991;101(suppl 53):1-78

4 Mantzouranis EC, Boikos SA, Chlouverakis G. Throat clearing - a novel asthma symptom in children. $N$ Engl J Med 2003;348:1502-3

5 Comings DE, Comings BG. Tourette syndrome: clinical and psychological aspects of 250 cases. Am J Hum Genet 1985;37:435-50

6 Butler DJ, Turkat NW. Great expectorations. A case of psychogenic throat clearing and expectoration. Arch Fam Med 1995;4:647-9

7 Lee B, Woo P. Chronic cough as a sign of laryngeal sensory neuropathy: diagnosis and treatment. Ann Otol Rhinol Laryngol 2005;114:253-7

8 Rawat AK. Throat clearing habit and ascariasis. Indian Pediatr 2001;38(suppl 9):1064

9 Irwin RS, Boulet LP, Cloutier MM, Fuller R, Gold PM, Hoffstein V et al. Managing cough as a defence mechanism and as a symptom - a consensus panel report of the American College of Chest Physicians. Chest 1998; 114(suppl 2):132-81

10 Quraishi MS, Jones NS, Mason J. The rheology of nasal mucus: a review. Clin Otolaryngol 1998;23:403-13

11 Boat TF, Cheng PW, Leigh MW. Biochemistry of mucus. In: Takishima T, Shimura S, eds. Airway Secretion: Lung Biology in Health and Disease. New York: Dekker, 1994; 217-82

12 Grossman J. One airway, one disease. Chest 1997; 111(suppl 2): $11-16 \mathrm{~S}$

13 O'Hara J, Jones NS. The aetiology of chronic cough: a review of current theories for the otorhinolaryngologist. J Laryngol Otol 2005;119:507-14

14 Sen P, Georgalas C, Bhattacharyya AK. A systematic review of the role of proton pump inhibitors for symptoms of laryngopharyngeal reflux. Clin Otolaryngol 2006;31: $20-4$

15 Carrau RL, Khidr A, Crawley JA, Hillson EM, Davis JK, Pashos CL. The impact of laryngopharyngeal reflux on patient-reported quality of life. Laryngoscope 2004;114: 670-4

16 Bhui K, Hotopf M. Somatization disorder. Br J Hosp Med 1997;58:145-9

17 Homer JJ, Sheard CE, Jones NS. Cognitive dissonance, the placebo effect and the evaluation of surgical results. Clin Otolaryngol 2000;25:195-9

Address for correspondence:

N S Jones,

Department of Otorhinolaryngology - Head and Neck

Surgery,

Queens Medical Centre University Hospital,

Nottingham University Hospitals NHS Trust,

Derby Road, Nottingham NG7 2UH, UK.

Fax: +44 1159709748

E-mail: nick.jones@nottingham.ac.uk

Mr A N Acharya takes responsibility for the integrity of the content of the paper.

Competing interests: None declared 